\title{
Universality of Brunnian ( $N$-body Borromean) four- and five-body systems
}

\author{
M. T. Yamashita \\ Instituto de Física Teórica, UNESP-Univ Estadual Paulista, C.P. 70532-2, CEP 01156-970 São Paulo, SP, Brazil
}

D. V. Fedorov and A. S. Jensen

Department of Physics and Astronomy, Aarhus University, DK-8000 Aarhus C, Denmark

(Received 9 November 2009; published 4 June 2010)

\begin{abstract}
We compute binding energies and root-mean-square radii for weakly bound systems of $N=4$ and 5 identical bosons. Ground and first excited states of an $N$-body system appear below the threshold for binding the system with $N-1$ particles. Their root-mean-square radii approach constants in the limit of weak binding. Their probability distributions are on average located in nonclassical regions of space which result in universal structures. Radii decrease with increasing particle number. The ground states for more than five particles are probably nonuniversal, whereas excited states may be universal.
\end{abstract}

DOI: 10.1103/PhysRevA.81.063607

PACS number(s): 03.75.Hh, 03.65.Ge, 03.75.Nt, 21.45.-v

\section{INTRODUCTION}

The Efimov effect was predicted in [1] as the appearance of a series of intimately related excited three-body states when at least two scattering lengths are infinitely large. These states can appear at all length scales and their properties are independent of the details of the potentials. This effect has in recent years been studied intensively and extended to a wider group of physical phenomena, beginning to be known as Efimov physics. In general, we define Efimov physics as quantum physics where universality and scale invariance apply. Universality means independence of the shape of the interparticle potential. Scale invariance means independence of the length scale of the system. These conditions are rather restrictive but a number of systems are known to exist within this window [2-7]. The great advantage is that one theory is sufficient to explain properties without any detailed knowledge of the interactions [8]. Furthermore, properties in different subfields of physics are described as manifestations of the same underlying theory.

Our physical definition of scale invariance originates from the halo physics first realized and discussed in nuclei [9], but quickly observed as applicable also to small molecules like the helium trimers [5]. This original definition of scale invariance, that the concept applies to any length scale, is obviously continuous as exemplified by nuclei, atoms, and molecules. Often the notion of scale invariance is used in a different mathematical sense, where the spatial extension of the structures in one given system repeats itself in discrete steps like the factor 22.7 for identical particles [1]. This is a result of the independence of potential details and here precisely defining our meaning with the notion of universality. Because the concepts can be defined in different ways, we use throughout the article this original physical meaning of scale invariance. Together, these two concepts constitute our meaning of Efimov physics.

The range of validity for such a global theory is only well described for two and three particles $[2,5,10]$. For $N=4$ two states were found in the zero-range, inherently universal, effective field model [11]. These states also appeared as universal in finite-range models in connection with each Efimov state [12]. This is in contrast to [13], where the disentanglement of the scales used to regularize the three and four-body zero-range Faddeev-Yakubovsky equations gives rise to a dependence of the four-body ground state on interaction details. Then a four-body scale is needed in analogy to the three-body scale appearing independently on top of the two-body properties. This apparent discrepancy between Refs. [11,12] and Ref. [13] is not yet resolved.

Recently, three experiments evidenced two four-body bound states connected to an Efimov trimer [14-16], in accordance with the theoretical predictions of Ref. [12]. In two of these experiments were also observed deviations from universality $[15,16]$. Surprisingly, the greatest deviations were observed for large scattering lengths $(\rightarrow \pm \infty)$ - exactly at the region where universality should apply [16]. This requires a theoretical explanation where something should be added in the universal model.

Very little is known for five particles with complete solutions containing all correlations as dictated by the interaction. With specific assumptions about only $s$ waves and essentially no correlations, it was concluded in [5,17] that ground-state halos cannot exist for $N>3$. These assumptions are rather extreme and could be wrong or only partly correct. However, if halos exist, they have universal structures as the $N=4$ states obtained in $[11,12]$. These results can only be reconciled by wrong assumptions in the halo discussion or by impermissible comparison between halo ground states and excited states.

It was concluded in [18] that Efimov states do not exist for $N>3$ and furthermore for three particles exist only for dimensions between 2.3 and 3.8 [19]. However, by restricting to two-body correlations within the $N$-body system, a series of (highly) excited $N$-body states were found with the characteristic Efimov scaling of energies and radii [20,21]. Whether they maintain their identity and the universal character when more correlations are allowed in the solutions remains to be seen.

Two limits to the universality are apparent. The first appears for large binding energy where the resulting small radii locate the system within the range of the potentials and sensitivity to details must appear. The less strict second limit is for excitation energies above the threshold for binding subsystems with fewer particles. Structures with such energies are necessarily 
continuum states which may, or more often may not, be classified as universal states depending on their structures and the final states reached after the decay.

Even for four particles where universality is found [11,12], a number of questions are still unanswered. For five and more particles the information becomes very scarce. A novel study claiming universality for ground states of a Van der Waals potential has appeared for particle numbers less than 40 [22]. The critical mass is found as a substitute for the critical strength, but the computed radii at threshold cannot be reliably extracted.

The purpose of the present article is to explore the window for Efimov physics. We investigate the boundaries for universality and preferably arrive at general conclusions applicable to systems of $N$-particles. We first discuss qualitative features and basic properties, then extract numerical results for four and five particles very close to thresholds of binding, and relate to classically allowed regions. We only investigate Brunnian ( $N$-body Borromean) systems [23] where no subsystem is bound.

\section{QUALITATIVE CONSIDERATIONS}

For two particles the infinite scattering length corresponds to a bound state at zero energy. Variation of $1 / a$ around zero produces either a bound state of spatial extension $a$ or a continuum state corresponding to spatial configurations correlated over the radius $a$.

For three particles the Efimov effect appears; that is, for the same interaction, $a= \pm \infty(1 / a=0)$, infinitely many three-body bound states emerge with progressively smaller binding and correspondingly larger radii [1]. The ratios of twoand three-body threshold strengths for several potentials were derived in $[22,24,25]$. These thresholds for binding one state can be characterized by a value of $1 / a[10,12]$. Infinitely many bound three-body states appear one by one as $1 / a$ is changed from the three-body threshold for binding to the threshold for two-body binding $1 / a=0$. Moving opposite by decreasing the attraction, these states, one by one, cease to be bound. They move into the continuum and continue as resonances [26]. For asymmetric systems with a bound two-body subsystem, the three-body bound state passes through the particle-dimer threshold, becoming a virtual state [27]. This behavior holds even for particles with different masses [28].

All three-body $s$-wave states from a certain energy and up are universal. However, this is not an a priori obvious conclusion, but is nevertheless true because two effects work together; that is, for $1 / a=0$ the system is large for the excited Efimov states and for finite $1 / a$ the binding is weak and the radius diverges with binding [5]. Both Efimov states and weakly bound states are much larger than the range of the interaction. The continuous connection of these bound states and resonances is therefore also in the universal region.

The recent results for four particles were that each threebody state has two four-body states attached with larger binding energy $[11,12]$. Both of these four-body states are described as having universal features unambiguously related to the corresponding three-body states for interactions of both positive and negative scattering lengths. Detailed information of structure, correlations, and posssible limits to universality are not available.

The one-to-one correspondence between the two four-body states and one three-body state can perhaps be extended such that two weakly bound $N$-body states appear below the ground state of the $(N-1)$-body state. This seems to be rather systematic for $N$-body Efimov states obtained with only two-body correlations [20,21]. If these $N$-body Efimov states remain after extension of the Hilbert space to allow all correlations, we can expect these sequences to be continued to the thresholds for binding by decreasing the attraction. However, ground and lowest excited states may be outside the universal region but the sequences may still exist. In any case, the scaling properties are different for the $N$-body Efimov states in $[20,21]$ and the universal four-body states in $[11,12]$.

The basic reason for the difficulties in finding detailed and general answers is related to the fact that the thresholds for binding are moving monotonously toward less attraction with $N$ [24,25]. For $N=2$ weak binding and large scattering length is synonymous. Already for $N=3$ this connection is broken but the weak binding still causes the size to diverge [5]. The indications are that for $N>3$ the size remains finite even in the limit of zero binding.

\section{BASIC PROPERTIES}

We consider a system of $N$ identical bosons each of mass $m$. They are confined by a harmonic trap of frequency $\omega_{t}$ corresponding to a length parameter $b_{t}^{2}=\hbar /\left(m \omega_{t}\right)$. The particles interact pairwise through a potential $V$ of short range $b \ll b_{t}$. We use the Gaussian shape $V=V_{0} \exp \left(-r^{2} / b^{2}\right)$. The chosen values of $V_{0}, b$, and $m$ lead to a two-body scattering length $a$ and an effective range $R_{e}$. The solution to the Schrödinger equation is approximately found by the stochastic variational method [29]. The results are energies and root-mean-square radii.

For two-body systems we know that the $n$th radial moment only diverges at threshold of binding when the angular momentum $l \leqslant(n+1) / 2$ (see [5]). The equality sign implies a logarithmic divergence with binding $B_{2}$ in contrast to the normal power law $B_{2}^{l-n / 2-1 / 2}$. For the mean square radius this implies divergence for $l \leqslant 3 / 2$. For an $N$-body system with all contributions entirely from $s$ waves we can generalize these rigorous results from two-body systems [5]. The number of degrees of freedom is $f=3(N-1)$ and the generalized centrifugal barrier is obtained with an effective angular momentum $l^{*}=(f-3) / 2$. Divergent root-mean-square radius is then expected when $l^{*} \leqslant 3 / 2$ or equivalently when $f \leqslant 6$ or $N \leqslant 3$. If this result holds, four-body systems should have finite root-mean-square radii even at the threshold of binding.

The size of the system is measured by the square root of the mean square radius, $\left\langle r^{2} / b^{2}\right\rangle$, which is expressed in units of the "natural" size of the systems, that is, the range of the binding potential. The dimensionless unit of the binding energy $B_{N}$ of the system is $\bar{B}=m b^{2}\left|B_{N}\right| / \hbar^{2}$. Both universality and scale invariance are therefore detected by inspection of these quantities as functions of parameters and shapes of the potentials. In regions where the curves are proportional, we conclude that the properties are universal and scale invariant. Results for different potential shapes can be expressed in terms 
of a standard potential by scaling the range. Then the individual curves would fall on top of each other in the universal regime.

\section{CLASSICAL ALLOWED REGION}

Universal properties can intuitively only appear when the structures are outside the potentials because otherwise any small modification would have an effect on the wave function. Consequently, the property would be dependent on these details in conflict with the assumption of universality. For two-body systems the relative wave function is therefore universal only if the largest probability is found outside the potential. This means that this classically forbidden region is occupied. The system is extremely quantum mechanical and very far from obeying the laws of classical physics.

To investigate the relation between universality and the classical forbidden regions for $N$ particles, we need to compare features of universality with occupation of classical forbidden regions. For two-body systems this is straightforward since the coordinate of the wave function and the potential is the same. The probability of finding the system where the energy is smaller than the potential energy is then easy to compute as a simple spatial integral over the absolute square of the wave function.

For more than two particles the problem is well defined but the classically forbidden regions (total energy is smaller than the potential energy) themselves are difficult to locate. We attempt a crude estimate which at best can only be valid on average. The energy is computed by adding kinetic and potential energy, that is,

$$
-B_{N}=N\left\langle t_{1}\right\rangle+\frac{1}{2} N(N-1)\left\langle V_{12}\right\rangle,
$$

where we choose an arbitrary particle 1 to get the kinetic part and a set of particles 1 and 2 to get the potential energy. The classical region is defined by having positive kinetic energy. For a two-body Gaussian potential we then obtain an estimate of an average, $r_{\mathrm{cl}}$, for the classical radius from

$$
\left\langle V_{12}\right\rangle>-\frac{2 B_{N}}{N(N-1)}=V_{0} \exp \left(-r_{\mathrm{cl}}^{2} / b^{2}\right) .
$$

If the distance between two particles is larger than $r_{\mathrm{cl}}$, we should be in the universal region. This value can then be compared to the size obtained from the average distance between two particles, $\left\langle r_{12}^{2}\right\rangle$, computed in the $N$-body system from the mean square radius [20]; that is,

$$
\left\langle\frac{r^{2}}{b^{2}}\right\rangle=\frac{N-1}{2 N}\left\langle\frac{r_{12}^{2}}{b^{2}}\right\rangle .
$$

Thus, in the classical forbidden region $r_{\mathrm{cl}}^{2} / b^{2}$ from Eq. (2) should be smaller than $\left\langle r_{12}^{2} / b^{2}\right\rangle$ from Eq. (3).

\section{THE FOUR-BODY SYSTEM}

We show size versus binding energy for $N=4$ in Fig. 1. The variation arises with change of the strength, $V_{0}$, of the attractive Gaussian. The system is for numerical convenience confined by an external one-body field. However, we are only interested in structures independent of that field, that is, intrinsic properties of the four-body system. We therefore increase the trap size until the states are converged and located

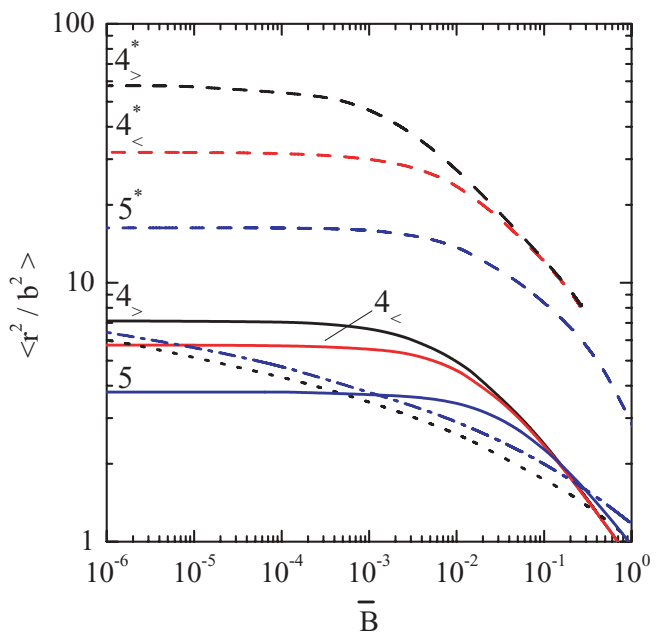

FIG. 1. (Color online) The mean square radius as function of the four- and five-body binding energies, all in dimensionless units. The trap sizes for four particles are $b_{t}=230.942 a_{0}$ (red lines with $4_{<}$), $2630.956 a_{0}$ (black lines with $4_{>}$), and $b_{t}=372.073 a_{0}$ (blue lines with 5 ) for five particles. Here $a_{0}$ is the Bohr radius. We show ground (solid) and excited (long-dashed with particle numbers tagged with an *) states and "classical" two-body radius [dotted (4) and dot-dashed (5)] translated by Eq. (3).

at distances much smaller than the confining walls. We now know that this happens for four particles in contrast to the three-body system, where the size diverges when the binding energy approaches zero.

In Fig. 1 we show results for two trap sizes deviating by an order of magnitude and larger than the interaction range $b=11.65 a_{0}$ ( $a_{0}$ is the Bohr radius) by a factor of 20 and 200 , respectively. For large binding in the bottom right corner the results for the ground state are independent of trap size. When the probability extends by about a factor of 2 further out than $b$ the effect of the small trap can be seen. The tail of the distribution then extends out to $20 b$ even though the mean square is 10 times smaller.

In the limit of very small binding energy the radius approaches a constant independent of the binding. The trap size has to be increased to $200 \mathrm{~b}$ before the trap has no influence, which implies that the probability distribution is entirely within that distance when the threshold for zero binding is reached. The converged size is about $2.7 b$ for the ground state. Somewhat surprisingly, also the first excited state, which also is below the energy of the three-body state, has converged to a value, $7.6 b$, independent of the trap size. A shape different from a Gaussian would again lead to constants related through specific properties of the potentials, but the ratio would remain unchanged. This is precisely as found in two dimensions for three particles [30]. Both states are at the threshold, on average, very much smaller than both traps. Nevertheless, the smallest trap would still influence the tail of the distribution.

In Fig. 1 we also show the estimated classical average distance between pairs of particles within the $N$-body system. This curve is above the ground-state radius for large binding. Here the probability is mostly found in the classical region within the potential, that is, in the nonuniversal region. Another potential shape would then move these curves. The classical 
and root-mean-square radius cross each other when the size is slightly larger than the range $b$. This limit for universality is similar to the halo condition for universality established in $[5,17]$. At smaller binding energy the classical radius becomes less than the size of the system and the probability is, on average, located outside the potential in the nonclassical, universal region.

For the extremely small binding energies close to the threshold, our estimate of the classical radius diverges logarithmically with binding energy. Thus, at some point it has to exceed the size of the system, which we concluded converges to a finite value for zero binding. This is simply due to the character of the Gaussian potential which approaches zero for large radii. Zero energy must then be matched by an infinite radius. However, this Gaussian tail is too small to obstruct the convergence of the probability distribution to a finite size. This cannot destroy universality because the tail has no influence on the wave function in this region far outside the range of the potential. For universality only the binding energy is decisive, as one can see explicitly for the two-body system. For $N$-body systems the same result follows from the asymptotic large distance behavior of the wave function expressed in hyperspherical coordinates [31]. Thus, the classical average radius argument fails for these extreme energies when the probability has settled outside the range of the short-range potential.

\section{FIVE-BODY SYSTEM}

In Fig. 1 we also show results for $N=5$, where convergence is reached for the trap size of $b_{t}=372 a_{0}$. We found two pentamers with energies $-0.0281 \frac{\hbar^{2}}{m b^{2}}$ and $-0.0113 \frac{\hbar^{2}}{m b^{2}}$ below the four-body threshold $\left(-0.0103 \frac{\hbar^{2}}{m b^{2}}\right)$ for infinite scattering length. The sizes for both ground and excited states increase again with decreasing binding energy $B_{5}$ and approach finite values when $B_{5}=0$. These limiting radii of about $1.94 \mathrm{~b}$ and $4.0 b$ are substantially smaller than corresponding values for four particles. Still, the largest probability is found outside the potential providing the binding. This strongly indicates that these structures are also in the universal region. Again, their ratio is anticipated to be essentially independent of potential shape. This conclusion is supported by the comparison in Fig. 1 to the classical radius, which always is smaller than the radius of the excited state and comparable to the radius of the ground state. As argued for four particles, the largest binding for the ground state corresponds to nonuniversal structure. When the binding energy is about 0.3 in the dimensionless units on the figure, the universal structure appears. This happens at about the same energy as for four particles. In both cases the probability is pushed outside the potential and universality is expected for smaller binding energies.

\section{CONCLUSIONS}

We have investigated the behavior of Brunnian systems near threshold for binding. Ground and first excited states for four and five identical bosons appear below the threshold for binding three and four particles, respectively. Their radii are for small binding energies larger than the range of the potential holding them together. The largest part of the probability is found in nonclassical regions, resulting in universal structures. For six and more particles the ground states would be located inside the potential and thus of nonuniversal structures. Excited states are larger and may still be universal, but already for seven or eight particles the first excited state is also expected to be nonuniversal. The numerical results are obtained for a two-body Gaussian potential but the features originating from wave functions in nonclassical regions of space are expected to be independent of the potential shape.

\section{ACKNOWLEDGMENTS}

We are grateful for helpful discussions with $\mathrm{M}$. Thøgersen. M.T.Y. thanks FAPESP and CNPq for partial support.
[1] V. Efimov, Phys. Lett. B 33, 563 (1970); Nat. Phys. 5, 533 (2009).

[2] K. Riisager, A. S. Jensen, and P. Møller, Nucl. Phys. A 548, 393 (1992).

[3] E. Nielsen, D. V. Fedorov, and A. S. Jensen, J. Phys. B 31, 4085 (1998).

[4] D. V. Fedorov, A. S. Jensen, and K. Riisager, Phys. Rev. C 49, 201 (1994); 50, 2372 (1994).

[5] A. S. Jensen, K. Riisager, D. V. Fedorov, and E. Garrido, Rev. Mod. Phys. 76, 215 (2004).

[6] M. T. Yamashita, T. Frederico, L. Tomio, and A. Delfino, Phys. Rev. A 68, 033406 (2003).

[7] M. T. Yamashita, R. S. Marques de Carvalho, L. Tomio, and T. Frederico, Phys. Rev. A 68, 012506 (2003).

[8] A. E. A. Amorim, T. Frederico, and L. Tomio, Phys. Rev. C 56, R2378 (1997).

[9] P. G. Hansen and B. Jonson, Europhys. Lett. 4, 409 (1987).

[10] E. Braaten and H.-W. Hammer, Phys. Rep. 428, 259 (2006).
[11] H. W. Hammer and L. Platter, Eur. Phys. J. A 32, 113 (2007)

[12] J. von Stecher, P. D'Incao, and C. H. Greene, Nat. Phys. 5, 417 (2009).

[13] M. T. Yamashita, L. Tomio, A. Delfino, and T. Frederico, Europhys. Lett. 75, 555 (2006).

[14] F. Ferlaino, S. Knoop, M. Berninger, W. Harm, J. P. D'Incao, H. C. Nagerl, and R. Grimm, Phys. Rev. Lett. 102, 140401 (2009).

[15] M. Zaccanti et al., Nat. Phys. 5, 586 (2009).

[16] S. E. Pollack, D. Dries, and R. G. Hulet, Science 326, 1683 (2009).

[17] K. Riisager, D. V. Fedorov, and A. S. Jensen, Europhys. Lett. 49, 547 (2000).

[18] R. D. Amado and J. V. Noble, Phys. Lett. B 35, 25 (1971); R. D. Amado and F. C. Greenwood, Phys. Rev. D 7, 2517 (1973).

[19] E. Nielsen, D. V. Fedorov, A. S. Jensen, and E. Garrido, Phys. Rep. 347, 373 (2001). 
[20] O. Sørensen, D. V. Fedorov, and A. S. Jensen, Phys. Rev. Lett. 89, 173002 (2002).

[21] M. Thøgersen, D. V. Fedorov, and A. S. Jensen, Europhys. Lett. 83, 30012 (2008).

[22] G. J. Hanna and D. Blume, Phys. Rev. A 74, 063604 (2006).

[23] H. Brunn, Über Verkettung, S.-B. Math.-Phys. Kl. Bayer Akad. Wiss. 22, 77 (1892).

[24] J.-M. Richard and S. Fleck, Phys. Rev. Lett. 73, 1464 (1994).

[25] J. Goy, J.-M. Richard, and S. Fleck, Phys. Rev. A 52, 3511 (1995).

[26] F. Bringas, M. T. Yamashita, and T. Frederico, Phys. Rev. A 69, 040702(R) (2004).
[27] M. T. Yamashita, T. Frederico, A. Delfino, and L. Tomio, Phys. Rev. A 66, 052702 (2002).

[28] M. T. Yamashita, T. Frederico, and L. Tomio, Phys. Rev. Lett. 99, 269201 (2007); Phys. Lett. B 660, 339 (2008).

[29] Y. Suzuki and K. Varga, Stochastic Variational Approach to Quantum-Mechanical Few-Body Problems (Springer, Berlin, 1998).

[30] E. Nielsen, D. V. Fedorov, and A. S. Jensen, Phys. Rev. A 56, 3287 (1997).

[31] S. P. Merkuriev, C. Gignoux, and A. Laverne, Ann. Phys. 99, 30 (1976). 